\title{
La production locale de médicaments génériques au Mozambique à la croisée des circulations de savoirs pharmaceutiques
}

Alila Brossard Antonielli

\section{OpenEdition}

\section{Journals}

Édition électronique

URL : http://journals.openedition.org/rfst/318

DOI : $10.4000 /$ rfst.318

ISSN : 2492-3672

Éditeur

Espaces et SOciétés (UMR 6590)

Référence électronique

Alila Brossard Antonielli, « La production locale de médicaments génériques au Mozambique à la croisée des circulations de savoirs pharmaceutiques », Revue francophone sur la santé et les territoires [En ligne], Les circulations en santé : des produits, des savoirs, des personnes en mouvement, mis en ligne le 16 décembre 2019, consulté le 06 avril 2021. URL : http://journals.openedition.org/rfst/318 ; DOI : https://doi.org/10.4000/rfst.318

Ce document a été généré automatiquement le 6 avril 2021.

\section{c) (1)}

La Revue francophone sur la santé et les territoires est mise à disposition selon les termes de la Licence Creative Commons Attribution - Pas d'Utilisation Commerciale - Partage dans les Mêmes Conditions 4.0 International. 


\title{
La production locale de médicaments génériques au Mozambique à la croisée des circulations de savoirs pharmaceutiques
}

\author{
Alila Brossard Antonielli
}

\section{Introduction}

1 Depuis une décennie, la production locale de médicaments en Afrique Subsaharienne a fait l'objet d'un nombre croissant d'études académiques et techniques. Ces travaux cherchent à comprendre quelles sont les conditions économiques, politiques et de savoirs nécessaires à l'installation et pérennisation d'industries locales de santé. L'enjeu étant principalement l'amélioration de l'accès aux médicaments pour les populations, mais également le développement de capacités en vue de diversifier l'économie. Le regain d'intérêt pour les industries pharmaceutiques en Afrique est une conséquence de l'introduction des Aspects des droits de propriété intellectuelle qui touchent au commerce (ADPIC) par l'OMC dans les années 1990. Les pays les moins avancés, dont une majorité en Afrique Subsaharienne, ont été jusqu'à présent dispensés d'adopter les brevets pharmaceutiques dans leurs législations nationales, et peuvent profiter de cette exception pour redévelopper la production locale de versions génériques de médicaments brevetés dans d'autres pays ${ }^{1}$ (Chaudhuri, Mackintosh et Mujinja, 2010 ; UNCTAD, 2011).

2 Si certains pays africains avaient des industries pharmaceutiques datant de la période coloniale - pour la plupart des filiales de firmes européennes - la production locale s'est surtout concrétisée après les indépendances à travers des politiques de développement industriel et de création de systèmes de santé nationaux. Plusieurs 
travaux recensent le recours des gouvernements et firmes privées africaines à diverses institutions internationales, aux investissements directs de firmes privées et à des experts pour débuter ou diversifier leur production de médicaments, majoritairement des génériques (Mackintosh et al., 2016; Pourraz, 2019). L'aide internationale pour promouvoir la production locale a pris des formes diverses dans le temps et selon les pays: financement de l'achat et de l'installation des équipements; transfert de technologie et des savoir-faire sur la production et garantie de qualité ; envoi d'experts pour le développement de nouvelles régulations, formation et soutien à l'obtention de certifications de qualité; et, plus récemment, création de marchés réservés, sous condition de respect des normes de qualité internationales (Chorev, 2019). À première vue, le déplacement de capitaux, de technologies et des personnes dans le domaine pharmaceutique sembler aller toujours vers l'Afrique, dans un mouvement à sens unique, mais un regard plus attentif des études de cas permet d'argumenter qu'il s'agit de circulations . En effet, au-delà du simple transfert d'un lieu à un autre, les expériences de production locale en Afrique donnent lieu à des mouvements récurrents d'allerretour. C'est le cas des personnes, que ce soit les experts internationaux se rendant dans divers pays au compte de l'UNIDO ou de l'OMS, ou des pharmaciens ou ingénieurs industriels africains (y compris de la diaspora indienne en Afrique) formés à l'étranger qui rentrent travailler dans leur pays d'origine ou ailleurs sur le continent, et dont les savoirs, les idées et les pratiques sont transformés par ces mouvements (Raj, 2013). De même, en ce qui concerne les modèles ou politiques en lien avec la production locale, la circulation implique des transformations, des traductions et adaptations par des acteurs et institutions ayant des objectifs et des ressources de pouvoir diverses (Porto de Oliveira et Faria, 2017). Cependant, le succès des initiatives de production locale de médicaments n'est pas le résultat naturel des circulations de technologies, machines ou experts. En effet, les firmes locales ont subi des difficultés à la suite des crises de la dette, des politiques d'ajustement structurel, mais également l'accroissement des exigences de régulations des produits pharmaceutiques (Quet, 2018), la rareté de main d'œuvre qualifiée et l'absence de politiques publiques coordonnant industrie et santé publique (Chaudhuri, Mackintosh et Mujinja, 2010).

3 Le cas du Mozambique s'inscrit doublement dans ces dynamiques, au passé comme au présent. La volonté affichée par le gouvernement à l'indépendance d'installer une usine pharmaceutique était, à l'instar de ses voisins, le prolongement d'une politique d'autonomie en médicaments essentiels s'inscrivant pleinement en dialogue avec les propositions l'OMS, de l'UNIDO et entre les pays Non Alignés dans la décennie 1970 (Patel, 1983). Si le Formulaire National de Médicaments, vitrine de cette politique, a été bien documenté à l'époque, les tentatives de démarrage de la production locale et les raisons des échecs ont été passées sous silence. D'autre part, la collaboration avec le Brésil pour l'installation d'une usine de médicaments génériques dans les années 2000 et l'arrivée d'une filiale d'une firme indienne sont des corollaires de la montée en puissance des industries pharmaceutiques des pays des Suds et des enjeux pour l'accès aux médicaments dans le contexte des ADPIC.

4 Cet article retrace les étapes des politiques pharmaceutiques au Mozambique et de la volonté de produire localement des médicaments essentiels. Bien avant la coopération avec le Brésil, celles-ci étaient traversées par des relations avec des institutions internationales et des circulations d'experts étrangers. Les tentatives de mise en place d'une industrie pharmaceutique locale, publiques et privées, au cours des 40 dernières années, relèvent à la fois d'enjeux locaux de santé et de l'inclusion de ces enjeux dans 
les agendas internationaux, des agences onusiennes comme de la diplomatie brésilienne. Nous essaierons de montrer comment les circulations d'experts étrangers et mozambicains n'aboutissent que partiellement à une acquisition et accumulation locale des capacités nécessaires à la production de médicaments. L'évolution du statut des médicaments au Mozambique illustre également la transformation de «Marx vers le marché » qui s'est opérée entre les politiques socialistes du pays après l'indépendance vers l'ajustement structurel et les privatisations des années 1990.

Cette recherche se base sur des entretiens et observations réalisés entre 2016 et 2019 au Mozambique et au Brésil auprès des acteurs politiques, des experts et techniciens travaillant pour la mise en place de l'industrie pharmaceutique et dans le renforcement de la Direction nationale de Pharmacie du Mozambique. Les entretiens ont été faits en langue portugaise, les extraits qui suivent sont traduits de façon libre par l'auteure, et la plupart des participants ont accepté d'être cités. Ces données empiriques sont complétées par l'analyse d'archives - télégrammes diplomatiques et rapports - issues du gouvernement brésilien et des institutions internationales, et une revue de presse 2 .

\section{Le Mozambique : champion des médicaments génériques à l'indépendance}

6 Depuis les années de lutte contre le colonisateur portugais, le droit à la santé pour toute la population était au cœur du projet politique et de développement de la FRELIMO (Front de libération du Mozambique), d'inspiration socialiste, qui prit le pouvoir à l'indépendance en 1975 (Mozambique, 1979; Walt et Cliff, 1986). Un mois avant l'indépendance, le futur ministre de la Santé du Mozambique s'était rendu à l'Assemblée mondiale de la santé de l'OMS où il participe aux discussions montantes sur les soins de santé primaires et sur les médicaments essentiels (Assemblée Mondiale de la Santé 28, 1975). Les politiques de santé mises en place au Mozambique indépendant s'inscrivaient pleinement dans les orientations de la déclaration d'Alma-Ata de 1978 (Mozambique, 1979; Walt et Cliff, 1986).

7 Parmi les aspects distinctifs de cette approche en santé, plusieurs mesures concernant l'organisation de l'achat et de la distribution de médicaments furent prises par le nouveau gouvernement dès les premiers mois de souveraineté. En septembre 1975, furent mises en place la Centrale de médicaments et d'articles médicaux (CMAM) et la Commission technique de Thérapeutique et Pharmacie (CTTF en portugais) (Barker, 1983 ; Velásquez, 1985).

8 La CMAM était chargée de centraliser le système d'acquisition, de stockage et de distribution des médicaments pour le service national de santé (SNS). La CTTF, composée de médecins, pharmaciens et pharmacologues mozambicains et étrangers, avait pour objectif d'élaborer une politique de médicaments, notamment à travers la création d'un formulaire national de médicaments qui listerait les produits nécessaires pour prévenir, diagnostiquer et traiter toutes les maladies existantes dans le pays et diffuser les informations scientifiques concernant l'usage et le choix des produits de santé (Martins, 1983). 


\section{Le Formulaire National des Médicaments : rationalisation socialiste en syntonie avec la liste médicaments essentiels de l'OMS}

Le premier Formulaire National de Médicaments (FNM), publié par décret du Ministère de la Santé (MISAU) le 25 janvier 1977, listait 640 produits, dont 314 médicaments, 18 sérums et vaccins, 14 produits d'anesthésie locale et générale, 62 formulations galéniques, 20 agents de diagnostic (Barker, 1983 ; Velásquez, 1985). Le Mozambique fut ainsi, avec le Sri Lanka, le Pakistan ou le Brésil, un des pays pionniers ayant devancé la liste de médicaments essentiels de l'OMS publiée en octobre 1977 (WHO, 1977). Les acteurs mozambicains de la santé ainsi que les auteurs ayant écrit sur la politique de médicaments mozambicaine ne se privent pas de souligner cette avance de quelques mois - tout en sachant que la démarche menant à la liste de médicaments essentiels était en cours depuis 1975 à l'OMS et que les principes en sont les mêmes (Greene, 2011; Marzagão et Segall, 1983). Cette politique fut dès le départ une vitrine du Mozambique indépendant.

10 Ce projet de limiter les médicaments disponibles dans le pays au minimum utile pour les maladies les plus courantes découlait également de l'expérience du premier ministre de la Santé du Mozambique H. Martins. Formé au Portugal, dont il déserte l'armée, il travaille en exil comme médecin rural et de santé publique au Maroc, en Algérie, puis en Tanzanie, et enfin comme expert scientifique dans la firme pharmaceutique suisse Roche. Il était convaincu que la majorité des produits pharmaceutiques étaient inutiles, existant uniquement pour produire des rendements à l'industrie. Selon Martins, l'approvisionnement, distribution et prescription de médicaments était «chaotique " pendant la période coloniale, avec un faible contrôle sur la qualité et la sûreté des 13000 médicaments et produits de santé alors en circulation. Des entreprises privées contrôlaient la totalité de l'importation et on recensait un représentant commercial pharmaceutique pour 5 médecins, exerçant principalement dans les villes, où étaient concentrées les pharmacies (Martins, 1983). Les médicaments étaient ainsi considérés pendant la période coloniale comme des marchandises, sans spécificité ni régulation particulière, soumises aux lois du marché et accessibles uniquement à ceux pouvant les payer (Velásquez, 1985). Dans ce contexte, la mise à disposition de médicaments pour l'ensemble de la population était une révolution selon Martins, mais une politique qui pour être viable devrait s'affranchir de la contrainte budgétaire.

11 Le choix des médicaments par la CTTF se faisait en fonction de leur efficacité thérapeutique et sûreté, mais également en prenant en compte le rapport coûtefficacité permettant au pays d'augmenter ses capacités d'acquisition de médicaments pour généraliser son assistance pharmaceutique avec un budget très restreint. Lorsque plusieurs médicaments étaient efficaces et sûrs pour une même maladie, la commission comparait alors les prix et choisissait le moins cher ou la forme médicamenteuse la plus adéquate et économique - les comprimés étant plus faciles à transporter et stocker que des ampoules ou liquides (Hanlon, 1983 ; Velásquez, 1985). Ainsi, sauf pour des raisons médicales prouvées, les médicaments sur la liste ne devraient pas «contenir des formulations galéniques exotiques, ni celles dont l'avantage n'est pas prouvé, ou celles trop chères ou inutiles " pour éviter le gaspillage de ressources (Martins, 1985).

12 Un des mécanismes de gestion rationalisée était l'adoption des noms génériques sur le formulaire national de médicaments. Cela permettait lors des achats faits par appel 
d'offres internationales du MISAU de changer de fournisseur en fonction des prix, pour s'affranchir des marques qui seraient rentrées dans les habitudes de prescriptions des médecins. La réduction du nombre de médicaments en circulation et la dénomination générique visaient à faciliter la prescription pour les médecins, mais aussi pour les infirmiers, assistants médicaux et paramédicaux, qui ont été autorisés à prescrire un nombre limité de médicaments du formulaire dans les centres de santé primaires à travers le pays, principalement en zone rurale. Le FNM se devait aussi d'être un instrument d'éducation pour les médecins et autres professionnels de santé (Barker, Marzagão et Segall, 1980). La CTTF élabora des Guides thérapeutiques qui accompagnaient le FNM avec des informations mises à jour sur les indications, posologies et effets secondaires des médicaments du formulaire. Ces guides faisaient mention des prix des médicaments et le rapport coût-efficacité des thérapies, une mesure prise pour que les professionnels prennent en considération l'impact économique de leurs prescriptions et ne recommandent que le strict nécessaire. En outre, le FNM visait à simplifier le contrôle des inventaires, le stockage, l'analyse et la distribution des produits pour relever le défi d'étendre la couverture médicale et pharmaceutique à l'ensemble du vaste territoire (Marzagão et Segall, 1983 ; Velásquez, 1985).

Figure 1. Article sur la politique pharmaceutique du Mozambique paru dans l'édition spéciale sur les médicaments essentiels du magazine de l'OMS Santé du Monde en juillet 1984.

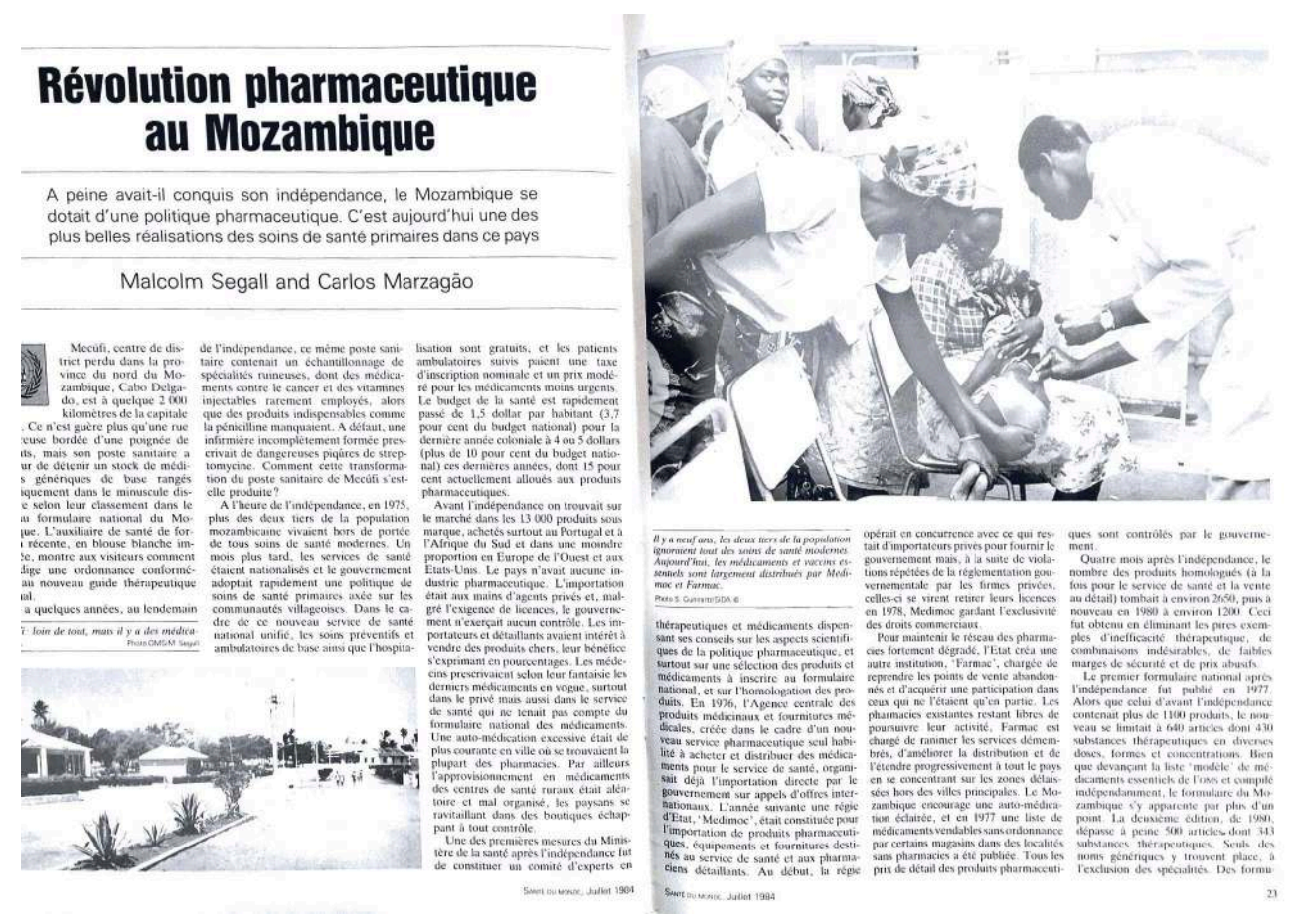

13 Ce nouveau système de rationalisation de la prescription fut complété par la création de l'entreprise d'État Medimoc en 1977 qui exécutait pour la CMAM l'achat et l'importation de médicaments à travers des appels d'offres internationaux. La comparaison entre les premières années d'indépendance, pendant lesquelles les entreprises d'importation privées continuaient de fonctionner et la transition vers le monopole de Medimoc en 1979 de toutes les importations de produits pharmaceutiques du pays, montre une réduction de $41 \%$ des coûts globaux d'importation pour le SNS (Velásquez, 1983). Le mode de distribution au sein du SNS permettait l'achat de 
médicaments en vrac et en formulations hospitalières, des formats moins onéreux que les emballages en petit nombre (Hanlon, 1983).

Le système d'achat par concurrence permettait la diversification des origines des importations, en provenance des pays socialistes - à hauteur de $40 \%$ en 1979, dont la Hongrie, la Bulgarie, la Chine, la RDA - mais aussi des pays occidentaux, le Portugal et la RFA en tête (Velásquez, 1985). Cette diversification apportait une sécurité dans l'approvisionnement et plus de choix en fonction des prix, mais impliquait davantage de pharmacovigilance pour tester localement la qualité des produits pharmaceutiques (Marzagão et Segall, 1983; Velásquez, 1983). Or, jusqu'en 1991 le Mozambique ne disposait pas d'un laboratoire de contrôle de médicaments et même lorsque celui-ci fut installé, ses capacités étaient dérisoires comparées au volume d'importations et des dons de médicaments qui affluaient d'Europe, des Amériques, d'Asie et de l'OMS. Selon les années et les catastrophes naturelles, les dons représentaient un tiers voire la moitié du total des médicaments importés et étaient parfois refusés lorsque les médicaments donnés ne figuraient pas dans la FNM (Hanlon, 1985).

Par ailleurs, de nombreuses publications furent faites à l'époque, par des membres du CTTF ou du MISAU en diverses langues, pour décrire et vanter les mérites économiques de cette nouvelle politique du médicament, d'autant plus extraordinaire étant donné les limites de ressources économiques et humaines. Le besoin pour ces acteurs de faire connaître et reconnaître cette nouvelle politique par le monde témoigne de l'ambition des acteurs politiques mozambicains et des coopérants étrangers travaillant au Mozambique ${ }^{3}$ d'inscrire leurs innovations politiques dans les débats mondiaux sur les médicaments essentiels à cette période. Ainsi, les autorités de santé du Mozambique étaient partie prenante dans les efforts politiques des pays du "Tiers-monde ${ }^{4}$ " pour défendre le modèle des listes de médicaments essentiels auprès des instances internationales, pour le légitimer et promouvoir son adoption par d'autres pays (Greene, 2011 ; OMS, 1979).

\section{Tour du monde à la recherche de la production locale de médicaments}

«Au cours des prochaines décennies, la mise en place de structures de soins de santé primaires dans les pays en développement devra s'accompagner du développement de moyens d'approvisionnement pharmaceutique, y compris de la production locale lorsque c'est possible, qui soient adaptés aux besoins réels de la majorité de la population. » (OMS Conseil Exécutif, 1978)

Les autorités mozambicaines auraient pu se contenter de faire des économies par l'achat groupé de médicaments et autres produits de santé. Mais elles avaient d'autres projets : la création d'une industrie pharmaceutique nationale pour développer une base technologique autonome dans le pays. "Nous devons créer, d'ici 1980, une industrie pharmaceutique capable d'assurer les besoins en produits essentiels, en utilisant progressivement plus de matières premières nationales ", affirmaient les Directives économiques et sociales du troisième congrès du FRELIMO (Moçambique, 1977). Velásquez (1985) estimait que la production locale permettrait d'économiser « de 30 à $40 \%$ par rapport au prix d'importation du produit fini » en devises, tout en rendant le Mozambique plus autonome. En outre, le développement des connaissances et des capacités technologiques et industrielles allait de pair avec les discours de 
développement moderniste du FRELIMO, en rupture avec la période coloniale qui avait faiblement investi dans l'industrie (Pitcher, 2002).

«J'ai toujours été favorable à la substitution des importations. Un des problèmes du Mozambique est [le] déficit de balance commerciale. Donc, quand j'ai voulu l'usine dans les années 70 c'était avec cet objectif; et deuxièmement à cette époque nous avions une vision socialiste et nous avions l'idée que tout devrait être d'État, les choses stratégiques devraient appartenir à l'État, et une usine de médicaments est une question stratégique. [...] en 1979, notre capacité technologique était bien inférieure à ce qu'elle est aujourd'hui, donc nous avions besoin de technologie étrangère [...] j'ai visité plusieurs pays socialistes à cette époque, la Yougoslavie en particulier et la Hongrie ; parce que la Hongrie était le pays au sein du Comecon ${ }^{5}$ qui était spécialisé en industrie pharmaceutique.» [H. Martins, médecin, ministre de la Santé du Mozambique 1975-1980]

Le Mozambique suivait alors l'exemple d'autres pays en développement ayant eu des politiques d'industrialisation et de diversification de leur économie juste après l'indépendance. L'Inde, le Kenya ou encore le Ghana dans les années 1950-60 faisaient appel à des investissements d'entreprises multinationales, mais aussi aux industries d'état des pays socialistes et au soutien des organisations internationales (Banda, Wangwe et Mackintosh, 2016; Pourraz, 2019 ; Quet, 2018). En ce sens, les acteurs de la santé du Mozambique ont également sollicité Cuba :

"L'objectif était d'aller voir localement les conditions opératives que les usines de médicaments exigeaient. Vois-tu, le type de ressources, le type de méthodologie, les formes d'organisation, les types de contrôles qu'ils faisaient. C'était donc une visite exploratoire pour que nous voyions comment cette industrie marchait et éventuellement ouvrir des chemins pour une collaboration future si nous avancions avec l'industrie pharmaceutique au Mozambique. Et cette visite de travail, c'était presque un mois à Cuba, était en lien avec l'organisme d'exportation de technologie de Cuba ». [J. Durão, économiste, directeur du Département Pharmaceutique, 1983-1989 et 1997-2005]

La coopération avec Cuba était logique dans la mesure où la révolution socialiste cubaine avait également mis en place un système de soins primaires et développait la recherche et la production de technologies en santé, en pharmacie et en biotechnologies, à travers des réseaux internationaux de coopération scientifique avec les pays socialistes et européens, comme la Suède et la France (Graber, 2016).

Les autorités mozambicaines ont également négocié l'installation d'une usine pharmaceutique sur leur territoire avec une firme pharmaceutique multinationale suédoise, Astra, et une étude de viabilité sera réalisée en 1979-1980, financée par le gouvernement suédois, un des soutiens de longue date du FRELIMO (Colin, 1976). Le gouvernement socialiste mozambicain accepterait de faire un "joint-venture " avec Astra, qui détiendrait $49 \%$ du capital de la future industrie et qui garderait la main sur l'administration et les cadres pendant les 10 premières années. Le projet prévoyait une production annuelle de 1000 millions de comprimés sous 33 formulations différentes et 2,5 millions de bouteilles de 15 formulations orales sous forme liquide (UNIDO, 1988a). Cependant, les discussions n'ont pas été poursuivies, car l'investissement prévu de 31 millions de dollars américains de l'époque, nécessaire pour l'achat d'équipements de haute technologie prévus par Astra, n'était pas réalisable. Les actions de déstabilisation économiques et armées, menées par l'Afrique du Sud sous régime d'Apartheid entre 1976-1984 et la Rhodésie du Sud sous le gouvernement d'Ian Smith entre 1976-79, puis la guerre civile mozambicaine, qui a duré jusqu'en 1992, ont accéléré la faillite économique du pays dans la décennie 1980 (Cahen, 1987). À cela s'ajoutaient les 
inondations (1977 et 1978) et les sécheresses (1982-83), mais également les conséquences de la mauvaise gestion de la planification et de la nationalisation de l'économie (Pitcher, 2002 ; Verschuur et al., 1986).

\section{La création ex nihilo de l'Entreprise Mozambicaine de Pharmacie (EMOFAR 1981-1988)}

20 À la demande du gouvernement mozambicain et malgré la détérioration de la situation économique du pays, une équipe hongroise se rend à Beira, au centre du Mozambique, à plusieurs reprises entre mai 1981 et avril 1983 pour installer une usine de sels de réhydratation orale (Kovacs, 1983). Malgré plusieurs problèmes liés à l'état des bâtiments et surtout aux coupures d'eau et d'électricité, l'équipe mandatée par l'UNIDO a testé un à un les équipements devant les autorités mozambicaines et élaboré les documents, en anglais et portugais, concernant les processus de production, les tests de contrôle en laboratoire, les manuels d'opération et d'entretien des équipements. Le 24 mars 1983, les experts hongrois lancent le test d'opération en continu des équipements, produisant 50000 poches de sels de réhydratation orale. Cependant, au moment des tests, l'usine ne disposait pas de personnel qualifié, mettant en doute la possibilité du fonctionnement continu de l'unité de production. À la fin de la mission, les autorités mozambicaines en accord avec le PNUD ont demandé à l'UNIDO de poursuivre son soutien en organisant des formations de ressources humaines. En conclusion de son rapport, le chef de la mission M. Kovacs «suggère qu'il serait possible de montrer l'usine aux experts de pays en développement qui ont l'objectif d'établir des usines similaires dans leur pays. "

21 Ainsi, dans un montage entre le PNUD, l'UNICEF et l'UNIDO comme exécutant, le Mozambique arrive à concrétiser une première usine de produits de santé, nommée EMOFAR (Empresa Moçambicana de Farmácia en portugais). Les sels de réhydratation orale étaient un produit peu complexe qui se prêtait bien à une introduction progressive de l'industrie pharmaceutique locale, comme prônée par le programme d'action en médicaments essentiels de l'OMS. Le choix du produit répondait également à des préoccupations de santé publique, les diarrhées infantiles et le choléra étaient un fléau au Mozambique. Ce produit pharmaceutique était un traitement efficace et particulièrement adapté à des contextes à ressources limitées; il s'agissait d'une innovation mise au point en Inde et au Bangladesh à la fin des années 1960 (Ruxin, 1994), puis promue par l'UNICEF à travers le « Tiers-monde ». Dans ce contexte, le choix de ce premier produit était ainsi un calcul entre les souhaits des autorités mozambicaines, l'agenda des institutions de santé internationales et les possibilités de financement de l'entreprise. Les capacités installées à EMOFAR étaient prévues pour produire 2 millions de sachets, soit $33 \%$ de la demande du Mozambique.

22 Le directeur du Département Pharmaceutique à partir de 1983 qualifie cette approche avec les institutions internationales comme plus pragmatique, par rapport aux tentatives précédentes négociées bilatéralement avec des pays socialistes ou Cuba :

« Ensuite, on est passé à un abordage plus terre à terre, dirais-je, en passant par une agence spécialisée des Nations Unies, l'UNIDO, qui se charge des choses de développement industriel. Ils ont embauché un spécialiste dans le domaine d'industrie pharmaceutique du Bangladesh, qui à cette époque était le pays qui se présentait comme un modèle alternatif aux grandes industries pharmaceutiques pour avoir une industrie fiable, de qualité, mais de faible coût opérationnel. [...]

Revue francophone sur la santé et les territoires, Les circulations en santé : des produits, des savoirs, des personnes en mouvement 
C'étaient des projets clé en main. C'est-à-dire, ces institutions donnaient le soutien au pays pour créer l'usine, formaient le personnel, faisaient le "trial run", et une fois prouvé que l'usine avait des conditions de fonctionnement, ils se retiraient et c'était la responsabilité du gouvernement de maintenir l'usine en fonctionnement.» [J. Durão, économiste, directeur du Département Pharmaceutique, 1983-1989 et 1997-2005] hongroise de l'UNIDO, de 1985 à 1987 un pharmacien industriel bangladeshi, A. Rahim, restera environ un an au Mozambique mandaté par la même agence. Il y effectuera plusieurs études pour la mise en place de la production de médicaments solides à EMOFAR, en commençant par une évaluation de la consommation de médicaments, le coût d'importation et la couverture par domaine thérapeutique (UNIDO, 1988a). Il a élaboré ensuite un plan en accord avec la consommation et les capacités techniques pour la mise en place d'une industrie pouvant produire 250 millions de comprimés en 15 formulations, 25 millions de gélules en 4 formulations et 1,85 millions de bouteilles de liquide oral. Selon lui, il était préférable de privilégier, dans un premier temps, la production de formes médicamenteuses simples en dosages non stériles, comme l'aspirine, la chloroquine ou le sulfate ferreux avec de l'acide folique. Il écarte la possibilité de production d'une large gamme de produits, notamment des pénicillines stériles, qui demanderaient des installations technologiques plus complexes, donc plus coûteuses en matériel et en formation de techniciens spécialisés. Le rapport relève également les capacités des industries auxiliaires locales, dont la plupart étaient des industries d'État, pour l'approvisionnement en verre (emballage primaire des médicaments) et en carton (emballage secondaire pour le transport). Il a été noté par exemple que les pots en verre étaient une solution moins onéreuse que l'achat de machines destinées à l'emballage en blister en plastique et aluminium qu'il faudrait importer.

nalement, Rahim a détaillé les besoins concernant la main-d'œuvre, les formations et l'expérience requises pour l'ensemble du personnel, estimé à 185 personnes, allant du directeur de l'usine aux opérateurs de production et de contrôle de qualité, en passant par les techniciens d'entretien des machines et le personnel administratif. Cependant, il souligne que le personnel en pharmacie ou en maintenance n'existait pas encore dans le pays et que les ministères de la Santé et de l'Éducation devraient prévoir la création de formations dans les divers domaines nécessaires à l'industrie. Ainsi, le plan prévoyait également le renfort avec l'aide internationale, agences de l'ONU et accords bilatéraux et régionaux, des capacités des laboratoires d'analyse et de contrôle de qualité, y compris par le développement de cours d'analyse de médicaments dans les programmes du département de chimie de l'Université Eduardo Mondlane de Maputo. Les agences de l'ONU avaient également le projet de soutenir l'Université dans la création d'une faculté de pharmacie, ce qui ne s'est pas concrétisé.

Les travaux d'adaptation et renforcement de la structure du bâtiment et des capacités électriques de l'EMOFAR ont lieu en 1987, ainsi que les premiers ateliers de formation de quatre responsables de production et de contrôle de qualité des médicaments (UNIDO, 1988b). La production d'EMOFAR en sels de réhydratation orale était à $25 \%$ de ses capacités en 1987 en raison de difficultés d'approvisionnement en glucose et en pièces de rechange pour les équipements. Cela entraînait une augmentation des coûts de production et rendait les importations de sels moins chères que la fabrication locale (World Bank, 1990). Nous n'avons pas pu savoir si l'usine a fonctionné à pleine capacité

Revue francophone sur la santé et les territoires, Les circulations en santé : des produits, des savoirs, des personnes en mouvement 
entre 1983 et 1987. Fin 1989, le gouvernement mozambicain était à la recherche de donateurs étrangers pouvant assurer un investissement de 250000 dollars américains de l'époque, afin d'augmenter la production de sels de réhydratation orale et de démarrer la production de comprimés. Le gouvernement venait de signer avec le Fonds Monétaire International et la Banque Mondiale un plan de restructuration et réforme économique. Par conséquent, l'État devait réduire ses dépenses et privatiser des entreprises publiques, comme Medimoc, ou créer des « joint-ventures» (Pitcher, 2002). L'EMOFAR n'a probablement pas trouvé de partenaire ou d'acheteur et, comme nombre d'entreprises étatiques à l'époque, elle a cessé ses activités entre 1988 et 1990. Dans les années 1990, le bâtiment d'EMOFAR, jouxtant celui de la Direction provinciale de la santé de la province de Sofala et de l'Hôpital Central de Beira, est annexé, la partie administrative est convertie en bureaux et la partie de stockage devient l'entrepôt provincial de médicaments.

27 Si la création ex nihilo de l'EMOFAR est issue de la volonté politique des acteurs mozambicains, elle se concrétise entièrement par l'intervention d'acteurs étrangers détenteurs de l'expertise et de financements. Les capacités d'investissement au sens de Lall (1992), c'est-à-dire l'identification des besoins, le dessin de la structure de l'usine, l'acquisition de la technologie et son installation sont le fait de l'équipe hongroise. Ces mêmes compétences de projection pour la partie de production de comprimés, complétée par le début de capacités de lien avec les industries auxiliaires, seront effectuées par le pharmacien bangladeshi. La formation de la main d'œuvre locale par les experts est restée très limitée, d'abord en nombre de personnels formés, mais également dans l'éventail de compétences de processus de production et de gestion. En effet, le nombre de pharmaciens mozambicains pouvant être formés dans la pharmacie industrielle à l'époque ne s'élevait pas à plus de cinq personnes, tous formés à l'étranger (Portugal et Cuba), et prenant à leur retour des responsabilités administratives à la CMAM ou à Medimoc. Le gouvernement mozambicain ayant décidé de créer uniquement une formation de niveau technique en pharmacie, il faudra attendre 1996 pour qu'une université privée ouvre un cursus supérieur en pharmacie dans le pays avec le soutien de professeurs de pharmacie du Portugal.

Dans un ouvrage sur l'expérience de la première décennie d'indépendance mozambicaine, Velásquez et d'autres coopérants internationaux concluaient en 1986 que le gouvernement mozambicain avait: «Un idéalisme et un manque de décision dans l'application de l'objectif de création d'une industrie pharmaceutique nationale [...]. L'inexistence d'une législation pharmaceutique complète fait que le fonctionnement de ce secteur dépend de façon exagérée de la capacité ou de la volonté de quelques personnes... » (Verschuur et al., 1986, p. 117-118). Ils soulignent l'ambition démesurée de l'installation de la production locale, qui était en décalage notoire avec les ressources économiques et les capacités humaines du pays à ce moment. Par la mise en valeur du bilan positif des économies en devises obtenues par le monopole de MEDIMOC et la critique des initiatives de production locale, les coopérants pointent vers le changement d'orientation des années 1990 qui priorisera les importations à moindre coût au détriment de la production locale. 


\section{« De Marx vers le marché » : la production locale de médicaments par des entreprises privées entre 1990 et 2004}

Swamo Indústria Farmacêutica (plus tard Petropharm) a ouvert en 1991 sous la propriété de deux entrepreneurs grecs. Mais dès sa première année, l'industrie n'arrive pas à vendre sa production de comprimés à Medimoc, qui ne disposait pas de fonds, malgré des ruptures de stock en médicaments partout dans le pays (Adamugy, 1992). De même, en 2000 des investisseurs mozambicains montent une usine de sérums de réhydratation intraveineuse à Matola, ville industrielle voisine de la capitale Maputo. Final Farmacêutica devait être fournie en équipements et connaissances par une entreprise sud-africaine, mais cette dernière fait faillite sans avoir complété les formations. Final avait embauché un pharmacien portugais et un technicien de maintenance zimbabwéen, ayant travaillé dans l'industrie pharmaceutique de son pays et des opérateurs de machines mozambicains.

"Quand Final commence ses activités, nous avions le Fonds Commun avec des règles très spécifiques, qui nous obligeaient à acheter au meilleur prix. La production de Final était, au minimum, le double du prix international. C'est-à-dire, avec l'argent du fonds il n'était pas possible d'acheter à Final; soit Final baissait son prix, en maintenant les standards [de qualité], c'est-à-dire elle serait en perte, et c'est à cette époque que commencent les premières discussions et divergences entre le gouvernement, des bailleurs de fonds ${ }^{6}$, [et le] Fonds Commun, etc., parce que le gouvernement a dû accommoder, disons ainsi, instituer quelques règles pour protéger la production locale [...] pour Final et aussi, par effet d'entraînement, des Grecs, qui à l'époque continuaient leurs activités. [...] Donc cela a amené à une gymnastique qui était d'essayer d'introduire dans le budget du Ministère de la Santé des fonds pour permettre de faire des achats locaux. Mais c'est un problème qui n'a jamais vraiment été résolu. » [J. Durão, économiste, directeur du Département Pharmaceutique, 1983-1989 et 1997-2005]

Bien que les informations disponibles sur ces deux expériences soient très limitées, il s'agit dans les deux cas d'entreprises centrées dans l'acquisition d'équipements sans le développement de compétences locales en production. Les cadres du MISAU soulignèrent que les investisseurs, qui n'avaient pas de liens avec le domaine de la santé, n'auraient pas suffisamment étudié le marché des médicaments dans le pays ni essayé de négocier au préalable avec le MISAU les possibilités d'achat pour la production locale. En plein dans la transition du socialisme vers l'économie capitaliste et sous ajustement structurel, le Mozambique était dépendant des ressources financières des bailleurs de fonds pour restructurer son système de santé et acheter des médicaments (Pavignani et Durão, 1999). Le gouvernement et les bailleurs mettent en place en 1995 un Fonds Commun pour l'approvisionnement des médicaments, financé entièrement par les donateurs et progressivement géré par le Ministère de la Santé Mozambicain, et dont l'objectif était l'optimisation des achats à moindre prix comme rappelé dans l'extrait ci-dessus. Par ailleurs, les deux entreprises ont subi des difficultés liées à l'augmentation des prix de matières premières et équipements et pièces de rechange importés (Pitcher, 2002). À la même époque, les crises de la dette ont entraîné la désindustrialisation des pays voisins comme la Tanzanie, notamment la fermeture d'industries pharmaceutiques (Banda, Wangwe et Mackintosh, 2016; Chaudhuri, Mackintosh et Mujinja, 2010). Petropharm et Final ont continué d'exister de façon claudicante jusqu'à la fin des années 2000, vendant leurs produits aux quelques pharmacies et hôpitaux privés ou au gouvernement lors de ruptures de stock. 


\title{
Le retour de la production locale : de la solution pour le $\mathrm{VIH} / \mathrm{SIDA}$ aux investissements des grands producteurs de génériques
}

31 L'enjeu pour l'accès aux traitements contre le $\mathrm{VIH} /$ sida, qui frappe $13 \%$ de la population adulte mozambicaine en 2001 selon l'ONUSIDA/OMS ${ }^{7}$, va raviver les espoirs de développer une production locale de médicaments. Dans son décret autorisant et régulant l'introduction des médicaments antirétroviraux (ARV) dans le pays, le MISAU, fidèle à ses principes d'accès aux médicaments essentiels génériques, va privilégier l'achat de ARV génériques. Le décret contient un paragraphe affirmant: « Le pays devra se préparer à produire, dans un futur proche, les médicaments en interne.» (Moçambique, 2001). Toutefois, le gouvernement admet dans ce texte ne pas avoir les moyens de couvrir l'assistance thérapeutique pour les personnes vivant avec le VIH, contrairement aux fondements du Service National de Santé du pays qui préconisait l'attention à toute la population. Ainsi, le décret précise que seuls les patients pouvant payer pour les examens de contrôle du virus pourraient accéder aux traitements. Cette affirmation de la volonté de mettre en place de la production locale paraît donc paradoxale, alors même que le pays n'a pas les moyens financiers pour garantir l'approvisionnement en ARV, ni les infrastructures ou le personnel pour les distribuer aux personnes qui en ont besoin ${ }^{8}$.

\section{L'offre de coopération brésilienne en $\mathrm{VIH} /$ sida et la demande mozambicaine en ARV}

\begin{abstract}
"Alors commença la discussion des antirétroviraux, à l'époque avec un prix exorbitant. À cette époque, le traitement coûtait environ 12000 dollars par an par malade. C'était inconcevable pour un pays africain de supporter ce coût. [...] On se rend compte que le Brésil est en train de produire ses propres médicaments, qu'il a adopté les mesures qui lui étaient permises dans le contexte des ADPIC et de la santé publique, pour pouvoir fabriquer ses propres médicaments et qu'il était en train de dispenser le traitement aux personnes les plus en besoin. C'était un service public. Et la technologie était là, avec des équipes reconnues, Farmanguinhos avait déjà de l'expérience démontrée en médicaments, donc nous [au Ministère de la Santé] avons dit "voyons, puisque nous avons toute cette discussion, nous sommes intéressés à coopérer avec le Brésil", ils étaient déjà là, ils formaient beaucoup de personnes au Brésil. Alors nous avons dit "pourquoi pas faire un pas de plus et commençons à concevoir une usine de médicaments pour résoudre ce problème", parce qu'on ne va pas dépendre du Brésil pour longtemps et le Brésil ne produisait pas beaucoup d'antirétroviraux à cette époque, même pour le marché interne le Brésil avait quelques problèmes. Alors nous avons dit que nous devions résoudre ce problème. » [F. Songane, médecin, ministre de la Santé du Mozambique 2000-2005]
\end{abstract}

En tant que ministre de la santé, F. Songane a participé en juillet 2000 à la conférence mondiale sur le sida à Durban, en Afrique du Sud. Il se souvient de manière enthousiaste de la mobilisation de la société civile sur place demandant l'accès aux traitements $\mathrm{du} \mathrm{VIH}$, dans le contexte du procès des multinationales pharmaceutiques pour empêcher l'Afrique du Sud d'importer des génériques. Dans cette même conférence, P. Teixeira, alors directeur du programme brésilien de lutte contre les IST/ $\mathrm{VIH} /$ sida, a annoncé que son gouvernement offrait aux pays africains la technologie et 
le savoir-faire acquis dans le principal laboratoire public de médicaments du pays, Farmanguinhos, qui approvisionnait le programme Sida brésilien depuis la moitié de la décennie 1990 pour une fraction du prix des ARV des multinationales (Scheinberg et Bouer, 2000). Le président brésilien, F.H.Cardoso, réitère cette offre dans les jours suivants à Maputo au Sommet de la Communauté des états de langue officielle portugaise (CPLP) (Cardoso, 2000). Les conférences mondiales du sida, ainsi que les rencontres des ministres de la Santé de la CPLP à l'OMS, furent citées par les cadres de santé mozambicains comme des lieux où ils rencontraient les responsables brésiliens de lutte contre le VIH/sida. L'expérience avérée et positive du Brésil dans le domaine et l'avantage de parler la même langue justifiaient la demande de soutien des Mozambicains ${ }^{9}$. Un premier protocole d'intentions en coopération en santé entre les deux pays sera signé en juin 2001 à Brasilia citant, parmi les domaines de coopération et d'échanges mutuels de savoirs, le «transfert de technologies dans le domaine de la production d'antirétroviraux, traitement et contrôle laboratoriel des personnes porteuses du VIH/sida » (Brasil/Moçambique, 2001).

La coopération démarre en 2002 avec le programme Ntwanano, dont un des volets incluait la formation de médecins mozambicains au Brésil et la venue des médecins brésiliens au Mozambique pour former leurs pairs en prise en charge du VIH, financé par la Fondation Ford jusqu'en 2005 (Lima, 2017; Luz, 2006). L'autre volet était l'inclusion du Mozambique dans le groupe d'une dizaine de pays africains et latinoaméricains qui recevaient des dons d'ARV permettant de traiter 100 patients par an, fabriqués au Brésil. Mais le directeur de Santé publique du MISAU considérait ce programme insuffisant au vu de l'ampleur de l'épidémie au Mozambique et demandait aux diplomates brésiliens l'élargissement de l'offre (Brasemb Maputo, 2002, 2003a). À l'issue de la rencontre du ministre de la santé mozambicain avec le ministre des affaires étrangères brésilien à Maputo en mai 2003, il n'est plus question du seul transfert de technologie, mais du soutien du Brésil pour construire une usine d'ARV. 
Figure 2. Le ministre de la Santé du Brésil, les présidents Lula da Silva, du Brésil, et Joaquim Chissano, du Mozambique, inspectent les antirétroviraux fournis par le gouvernement brésilien à I'Hôpital Central de Maputo en novembre 2003. Photo : R. Stuckert/Reuteurs

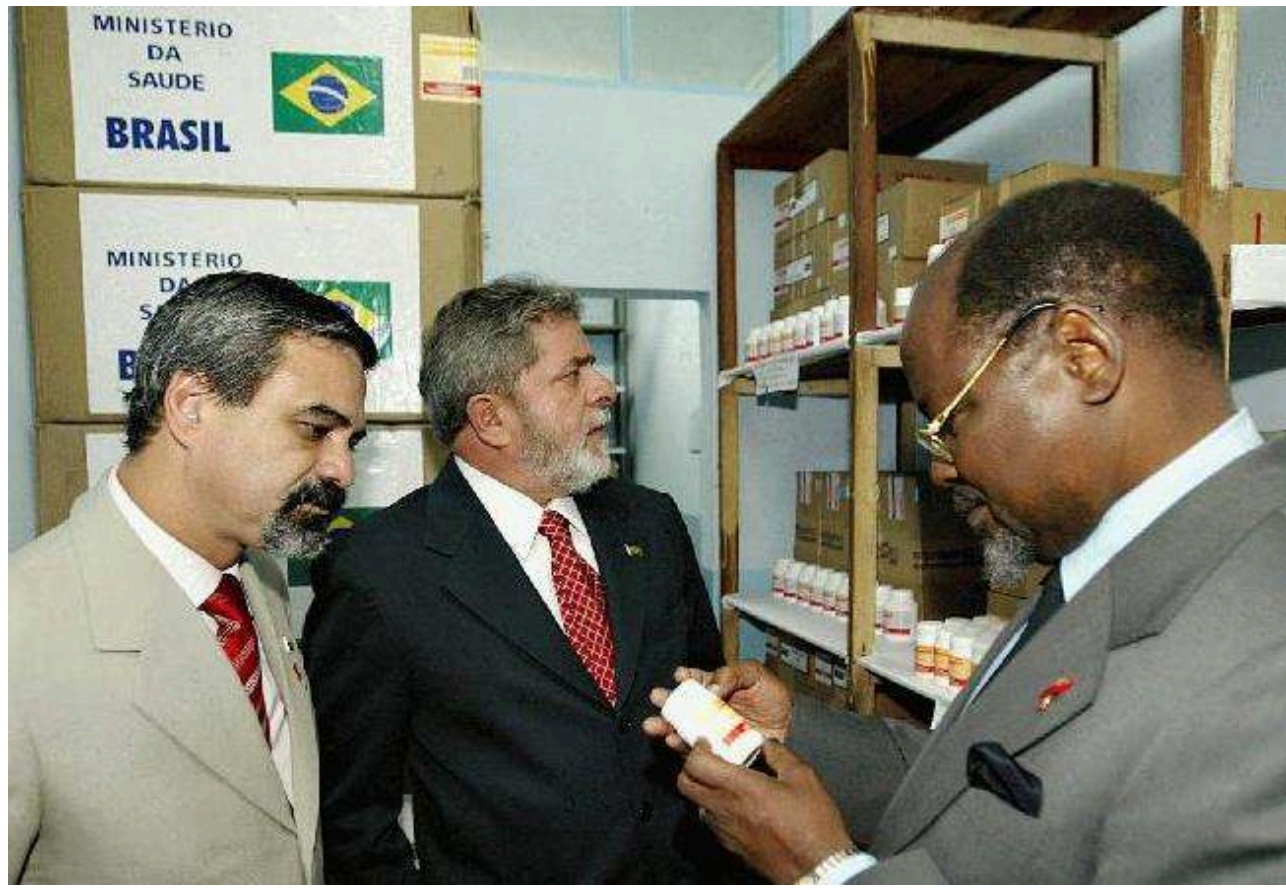

Proposée comme l'étape suivante de cette coopération, l'usine de médicaments génériques serait, dans la vision partagée par les Brésiliens et les Mozambicains au début des années 2000 , la solution pérenne pour le problème d'accès aux traitements. En effet, du côté brésilien ce projet répondait aux principes de la coopération sud-sud structurante en santé proposée par les acteurs de la Fiocruz - fondation de recherche sur la santé publique du Ministère de la santé brésilien - qui mettait l'accent sur la formation des ressources humaines, le transfert et l'adaptation des connaissances aux besoins des pays récepteurs et le renforcement des structures sanitaires, y compris de la production de biens en santé (Almeida et al., 2010 ; Russo et al., 2014). La coopération brésilienne en matière de $\mathrm{VIH} /$ sida faisait également partie d'une stratégie de défense de la politique nationale de traitement au moment de la remise en question de la loi brésilienne de brevets à l'OMC au début des années 2000 (Flynn, 2013). Elle sera progressivement intégrée dans l'éventail d'outils de la politique étrangère brésilienne pour renforcer les liens avec les pays du Sud.

\section{La Société Mozambicaine de Médicaments : une usine nationale co- créée avec le Brésil}

35 Une mission d'experts pharmaciens de Farmaguinhos s'est rendue au Mozambique en juillet 2003 pour relever les conditions pour l'installation d'une usine, visitant les locaux de Final et de Petropharm ainsi que la seule faculté de pharmacie (Brasemb Maputo, 2003b). L'accord de coopération spécifique à l'installation de l'usine d'antirétroviraux est signé en novembre au moment de la visite officielle du Président Lula da Silva à Maputo (Brasil/Moçambique, 2003).

36 Le Brésil assurait les formations des ressources humaines pour la production et la gestion du laboratoire et le Mozambique s'engageait à mettre à disposition une 
structure physique pour l'installation du laboratoire, identifier des ressources humaines à former, veiller à la diffusion des connaissances auprès d'autres techniciens nationaux et fournir les matières nécessaires au fonctionnement et à la production des médicaments. Le financement du projet, estimé à 23 millions de dollars, devait majoritairement être assuré par l'État brésilien, incluant l'achat des équipements pour la production et le laboratoire de contrôle de la qualité. Le gouvernement mozambicain a acquis les locaux de Final pour un montant non précisé en 2008 et l'a constitué en société privée, la Société Mozambicaine de Médicaments (SMM) dont le capital appartient $100 \%$ à l'État mozambicain, géré par l'Institut de Participations de l'État (IGEPE), sous tutelle du Ministère des Finances. La compagnie minière brésilienne Vale, installée au Mozambique, a comblé le manque de ressources de l'État mozambicain pour les travaux d'adaptation du bâtiment en 2011 à hauteur de 4,5 millions de dollars (Rodrigues 2014).

Une étude technique et économique sera menée par des experts de la Fiocruz entre 2005 et 2007, avec des résultats controversés en ce qui concerne les projections des médicaments à fabriquer et la viabilité économique dans le nouveau contexte des bailleurs de fonds internationaux (FIOCRUZ, 2007). En effet, à partir de 2004 le PEPFAR (United States President's Emergency Program for AIDS Relief) assure le financement et la distribution de deux tiers à trois quarts des médicaments ARV distribués (selon les années) au Mozambique (Pfeiffer, 2013). Ces ARV sont achetés pour l'ensemble des pays bénéficiaires du programme à travers des appels d'offres internationaux ouverts à des industries pharmaceutiques ayant les certifications de qualité de la Food and Drug Administration des États-Unis ou la préqualification de l'OMS. Se dessinait ainsi une situation semblable à celle vécue par les entreprises pharmaceutiques installées au Mozambique auparavant ou celles des industries pharmaceutiques ailleurs en Afrique, où la production locale ne peut pas rentrer dans les marchés des donateurs mondiaux qui opèrent selon des critères qui les excluent (Banda, Wangwe et Mackintosh, 2016 ; Cassier, 2019).

Parallèlement, les ministres successifs de la Santé du Mozambique demanderont au Brésil d'élargir le portfolio des médicaments de la future usine pour inclure, en plus des ARV, d'autres médicaments essentiels, ce qui permettra la continuité du projet.

«Pendant le processus de négociation, une fois les comptes faits avec les techniciens, nous avons constaté que la capacité de production installée était trop importante pour ne produire que des ARV [...]. De là l'idée est venue que, comme le Mozambique n'a aucune industrie pharmaceutique, la même usine puisse être utilisée pour produire d'autres médicaments ayant une grande demande dans le pays et ainsi l'usine serait plus rentable. Nous avons détaillé les négociations et nous avons fait une liste de 21 autres médicaments qui seraient produits. Il s'agit au fond de la liste des médicaments les plus demandés et sur lesquels le gouvernement dépense beaucoup d'argent à importer. [...] Nous voulions une usine du gouvernement du Mozambique pour produire non seulement des ARV, mais aussi 21 médicaments que nous allions vendre à un prix extrêmement bas, qui seraient gratuits dans le service national de santé. [...] les Brésiliens ont dit "non, il n'y a pas de problème [de changer le projet pour inclure d'autres médicaments génériques en plus des ARV].» [P.I. Garrido, médecin, ministre de la Santé du Mozambique 2005-2010]

L'installation de la production locale avec le soutien de la coopération brésilienne s'inscrivait dans la continuité de la politique pharmaceutique de l'indépendance mozambicaine. Le discours d'autonomie d'un pays du sud vis-à-vis des laboratoires 
pharmaceutiques multinationaux sera repris par la communication du gouvernement brésilien et en particulier par le président Lula, dont le Parti des Travailleurs a des liens anciens avec le FRELIMO (Hervieu, 2011). Les autorités de santé mozambicaines insistaient également que le projet devait être économiquement autonome et dès l'annonce souhaitent que l'usine fût orientée pour exporter des ARV et d'autres médicaments vers l'Afrique australe (Notícias, 2003). Cela a mené à des « malentendus » entre les acteurs brésiliens, qui conçoivent la SMM comme un miroir de Farmanguinhos, exclusivement tournée vers les besoins de santé nationaux et sans but lucratif et l'IGEPE pour qui la priorité est la rentabilité de la SMM.

La liste des médicaments devant être produits a fréquemment évolué de 2007 à 2017 en fonction des possibilités de fabrication de l'usine telle qu'installée, des changements des recommandations de traitement, notamment des ARV et de l'évolution de leur demande et de leur prescription dans le système national de santé. À ce jour, l'usine a produit des lots tests de nevirapine (ARV) en 2013, propanolol et captopril (antihypertensifs), halopéridol (antipsychotique), glibenclamide (anti-diabétique) et de l'acide folique entre 2014 et 2016. La SMM importe, à la demande du MISAU, de l'amoxicilline (antibiotique), fabriquée à Farmanguinhos mais enregistrée sous marque SMM au Mozambique. L'inclusion récente en 2017 du paracétamol (antalgique et antipyrétique), un médicament qui contrairement aux précédents n'est pas produit par Farmanguinhos, a répondu à des critères liés à la demande du MISAU. Il s'agit du médicament le plus consommé en volume dans le pays, permettant de faire des économies d'échelle et de répondre aux besoins de rentabilité économique de l'usine.

\section{Entre théorie et pratique : l'apprentissage technologique à la SMM}

Farmanguinhos devient l'institution responsable de la mise en œuvre du projet en 2008 et embauche une équipe d'une dizaine de pharmaciens, chimistes et ingénieurs spécialisés dans les équipements de production pharmaceutique. Cette équipe est issue des rangs des industries pharmaceutiques privées au Brésil, nationales et multinationales. Il ne s'agit donc pas du personnel ayant fabriqué des ARV dans l'usine de Farmanguinhos au Brésil et qui transmet leurs savoirs au personnel de la SMM, mais des professionnels qui partagent l'ensemble de leurs compétences et leur expérience en production, en garantie de qualité et en inspection dans plusieurs entreprises privées à la SMM. L'embauche de techniciens et cadres locaux a été problématique, car aucun des pharmaciens mozambicains ne disposait d'expérience en industrie pharmaceutique. Ceux ayant travaillé à EMOFAR n'auraient pas, à notre connaissance, été mobilisés par le MISAU pour relancer la production locale, marquant l'absence de cumul de l'expérience précédente.

«De manière générale, il n'existe pas d'industrie pharmaceutique au Mozambique. Il y a des pharmaciens, oui, ils sont peu nombreux, mais il y en a et sans aucune connaissance de l'industrie pharmaceutique. Je ne parle pas que des pharmaciens, mais de tous les domaines [de l'usine]. Donc lorsqu'on reçoit un nouveau collègue c'est la question de former, de l'entrainer, nous devons lui apprendre tout de zéro ; et si c'est déjà un défi pour le personnel qui est déjà ici depuis un moment de mettre en œuvre les bonnes pratiques de fabrication, imagine ce que c'est d'inculquer cela à quelqu'un qui vient de l'extérieur, nouveau et qui commence de zéro. Et le défi c'est que souvent on les forme, ce n'est pas facile de former les personnes dans un domaine aussi spécifique, et la crainte que la personne soit bien formée et qu'elle reste ici [rires] ! Si la personne disparait, c'est un autre scandale, 
c'est cent pas en arrière. [Nous avons eu] beaucoup de départs, de fuite de cadres. » [Pharmacienne mozambicaine, responsable du service de garantie de la qualité à la SMM de 2012 à 2018] au Brésil à partir de 2011. Ils suivront des formations intensives à Farmanguinhos d'une durée d'une semaine à un mois, à la fois théoriques, puis pratiques en s'immergeant dans la production sur place. Dans l'autre sens, les experts brésiliens de Farmanguinhos se rendent à intervalles réguliers à la SMM pour des missions de formation « on-the-job » des 55 employés. Le travail d'installation des machines, de contrôle de qualité et de la mise en place des documents de garantie de qualité est réalisé progressivement par un technicien mozambicain sous la supervision de son binôme brésilien (Araujo, Duarte et De Castro, 2017; Rodrigues, 2014). Les compétences transmises sont les capacités élémentaires de production, soit l'acquisition par répétition de l'ingénierie des processus avant, pendant et après la production (calibrer les équipements et l'environnement de production, contrôles de qualité) et l'ingénierie des produits (pesage, mélange des matières premières, compressage, emballage) (Lall, 1992). technologies, mais de l'ensemble de dispositions, savoirs et pratiques de gestion d'une industrie pharmaceutique, dans le contexte très concurrentiel du marché mondial de médicaments régi par des normes internationales comme les Bonnes pratiques de fabrication (BPF) (Quet, 2018). Les BPF sont des normes mises en place d'abord par les agences de régulation des médicaments des pays développés, puis diffusées à travers les industries des pays du Sud notamment par les exigences de certification internationales, pour standardiser la production de médicaments, en garantir la qualité et sûreté. Le respect et la certification en BPF impliquent de nombreuses heures de formation, certaines renouvelées obligatoirement tous les ans, et la mise en place d'un système rigoureux d'enregistrement uniformisé de toutes les procédures de fabrication. En outre, la formation des cadres de la SMM en gestion et en direction de production a inclus récemment des voyages à une foire internationale de pharmacie en Allemagne en 2017 et des visites à des fournisseurs et potentiels partenaires en Chine en 2018, accompagnés par une partie de l'équipe de Farmanguinhos.

«Une chose c'est de parler de technologie d'un équipement, etc., autre chose c'est de monter un négoce pharmaceutique, dont la technologie fait partie. On parle de structurer une industrie avec divers domaines de connaissances, avec un profil de professionnels principalement orientés vers la partie pharmaceutique et chimique qui n'existait pas dans le pays. Et là, oui, avoir une structure technologique montée et les gens qui apprennent non la technologie de ce médicament, mais à opérer une industrie pharmaceutique [...] l'objet qui était de construire une industrie pharmaceutique, faire un transfert de technologie vers un autre pays, la seule hypothèse qu'a cette usine pour survivre c'est qu'elle réponde aux critères internationaux, parce qu'autrement elle était condamnée à l'échec. » [L. de Oliveira, pharmacienne, directrice de la coopération Farmanguinhos-SMM depuis 2008]

Contrairement aux expériences des usines privées, le projet mozambico-brésilien insiste sur l'apprentissage technique au sens de «l'ensemble des expériences, discontinues ou fragmentées, au sein des systèmes productifs qui permettent le développement des pratiques et des savoirs nécessaires au fonctionnement et au maintien de ces systèmes pour les entreprises » (Arvanitis, Villavicencio et Wei, 2014). L'assimilation des savoirs organisationnels et technologiques pour la production se fait ainsi majoritairement par des dispositifs explicites de formation, avec des évaluations

Revue francophone sur la santé et les territoires, Les circulations en santé : des produits, des savoirs, des personnes en mouvement 
du personnel formé enregistrées dans les documents du service de garantie de la qualité de la SMM. De plus, dans les rapports des missions, les acteurs brésiliens comptabilisent également comme formation toutes les heures des activités de routine réalisées en binôme au sein l'usine, soulignant ainsi l'importance de l'assimilation de savoirs tacites et la formation « sur le tas».

Tout en reconnaissant l'importance de ces formations, de leur stabilité et continuité sur le long terme - la coopération dure depuis plus de dix ans, malgré quelques mois de suspension pour des raisons budgétaires-, les Mozambicains et les Brésiliens soulignent la difficulté d'absorption des connaissances. Ils constatent un énorme fossé de savoirs, de savoir-faire tacites et de connaissances des processus de planification et de production. L'idéal du transfert horizontal des connaissances entre des professionnels issus de pays en développement, même à niveau de formation supérieure égale, ne résiste pas à l'asymétrie des compétences et d'expérience professionnelle dans l'industrie pharmaceutique (Esteves et Assunção, 2017). L'accumulation à long terme de cette formation pose problème, comme dans d'autres industries pharmaceutiques africaines, du fait de la difficulté de garder la maind'œuvre formée, attirée par des offres d'emplois mieux rémunérés dans le privé ou auprès des ONG internationales (Russo et Banda, 2015). Aussi, si l'apprentissage technologique se concrétise par la pratique, l'absence de production en continu - la production de comprimés a été complètement interrompue d'avril 2016 à mars 2018 en raison de l'interruption des financements brésiliens et de la crise des dettes secrètes mozambicaines ${ }^{10}$ - limite la maitrise des savoir-faire par le personnel de l'usine.

La spécificité de la SMM, à la fois une industrie publique et soutenue par un gouvernement étranger, implique une longue série de contraintes administratives, budgétaires et politiques, soumise à des négociations entre les bureaucraties de pays ayant des visions de politiques publiques différentes et livrées aux aléas des changements de gouvernement et des crises économiques qui traversent le Brésil et le Mozambique depuis 2016 (Abdenur et Marcondes, 2017 ; Esteves et Assunção, 2017 ; Russo et de Oliveira, 2016). Les règles pour les entreprises d'État de l'Institut de Participations de l'État (IGEPE) exigent que celles-ci soient rentables, mais ne fournissent pas de capital pour le départ de l'activité, ce qui a mis la SMM en difficultés depuis son démarrage.

Lorsqu'il achète la production de la SMM ou l'importation d'amoxicilline, le MISAU met souvent plusieurs mois à payer, ce qui se traduit par des retards de salaires ou de paiement de factures à la SMM. L'entreprise a aussi connu des problèmes de fournisseurs et de dédouanement qui ont fortement retardé ses activités (Barbier, 2014).

Ainsi, plusieurs acteurs au sein du gouvernement mozambicain, y compris au MISAU, préfèreraient que l'entreprise devienne un "joint-venture » ou soit entièrement privatisée et voient l'exportation comme l'objectif ultime de l'initiative. Si le Ministère de l'Industrie et du Commerce mozambicain a lancé un plan stratégique industriel et la promotion du "Made in Mozambique », les responsables du programme ignoraient il y a encore quelques mois l'existence de deux usines de médicaments dans leur pays (ils en ont été informés à la suite des entretiens réalisés dans le cadre de cette recherche). Ces cadres sont peu nombreux pour un travail gargantuesque de coordination des investissements privés, qu'ils attendent surtout dans des domaines à haut rendement tels que l'extraction de ressources minières. Ils sont peu coordonnés avec les cadres du 
MISAU, qui à leur tour semblent être désinvestis de la SMM, très rarement mentionnée dans les discours ou les documents relatifs à la santé. du projet de coopération le plus long et le plus coûteux en ressources - et certains cadres mozambicains souhaitent que la SMM reste orientée en priorité aux objectifs de santé publique, mais sont ouverts à des partenariats privés. En ce sens, ces derniers travaillent à l'autonomie de la SMM par rapport à Farmanguinhos. Cela se traduit par l'exploration de partenariats sur des génériques spécifiques avec des entreprises multinationales; l'adhésion de la SMM en 2018 à l'Association de producteurs de médicaments génériques de l'Afrique australe (SAGMA); et la mise en bourse de l'entreprise en 2018.

\section{Les ambivalences de la concurrence indienne}

En 2016, une grande firme indienne privée de génériques, Strides, s'installe à un quart d'heure de route de la SMM à Matola avec deux lignes de productions de génériques, dont une pour des antibiotiques. Début 2017, elle envoie une dizaine de Mozambicains, dont certains issus des rangs de la SMM, en formation pendant deux mois dans les usines de la maison mère en Inde. À leur retour, les Mozambicains, assistés des directeurs indiens, soumettront l'ensemble de la documentation pour la certification de l'usine par les autorités mozambicaines. Elle a été inspectée et certifiée en octobre 2017, en même temps que la SMM, par une consultante de l'OMS pour le Département Pharmaceutique. Pour l'instant, certains produits fabriqués sur place ont déjà été en partie vendus au MISAU, mais leur autorisation de mise sur le marché reste soumise à la conclusion d'études de stabilité des médicaments sur deux ans menés par l'entreprise.

51 Le Département Pharmaceutique voit d'un bon œil cette nouvelle entreprise, soulignant les impressionnantes capacités de production installées, qui dépasseraient les besoins du Mozambique et indiqueraient la propension à exporter vers l'Afrique australe. Toutefois, selon des pharmaciens, les salaires payés par Strides sont peu attractifs, impliquant un risque de rotation des cadres et de faible développement de compétences locales. En effet, bien qu'ayant formé des Mozambicains, l'entreprise s'appuie davantage sur les compétences de gestion et exécution de la production des employés expatriés indiens au sommet de la hiérarchie de l'usine. De même, l'ensemble des procédés et normes de production sont importés de l'usine mère indienne, qui ne transfère que le nécessaire pour la production des génériques. Ainsi, les employés mozambicains sont cantonnés à des activités d'exécution et uniquement dans les processus de production et de contrôle de qualité, limitant la capacité d'apprentissage technologique local. Les pharmaciens mozambicains se soucient également de la capacité de leur pays à réguler l'entreprise :

«Dans les industries, des erreurs peuvent avoir lieu et le produit est dispensé à la population qui immédiatement le consomme, parce qu'il n'y a pas de contrôle. Nous avons livré maintenant au Ministère de la Santé, et je pense que... qu'ils ne sont pas, ... avant de recevoir ils doivent comprendre comment fonctionne une ligne de production de ce médicament qu'ils reçoivent. Donc, si nous ici, le département en lui-même, n'est pas en capacité de savoir quels sont les limites acceptables d'une production, d'un médicament spécifique, ils vont accepter ce qu'on leur présente. [...] il devrait y avoir un contrôle très profond des médicaments. Ce qui se passe c'est qu'aucun organe ne le voit, même s'ils sont vraiment préoccupés à vouloir des

Revue francophone sur la santé et les territoires, Les circulations en santé : des produits, des savoirs, des personnes en mouvement 
produits de qualité, mais jusqu'à quel point ils sont de qualité, jusqu'à quel point? Parce que d'un côté c'est l'industrie, le business, il faut faire du profit ; l'autre côté, il y a la qualité du produit que la population consomme, peut-être qu'au lieu de guérir il ne fait pas plus de dégâts?»

52 Cet employé de Strides, qui a demandé à rester anonyme, souligne en entretien la pression constante, dès la formation en Inde, à travailler en cadence accélérée pour rentabiliser et témoigne de moments où les règles de garantie de qualité n'auraient pas été entièrement respectées. Sans y faire allusion directement, il relaie des craintes à l'échelle mondiale contre les industries pharmaceutiques du sud perçues comme moins regardantes des normes de qualités (Quet, 2018). Cela serait dû en partie par l'effet de concurrence de réduire les coûts de production et de la moindre capacité, par manque de personnel qualifié et de moyens, des autorités de contrôle et de régulation de faire respecter les normes.

\section{Conclusion}

53 En retraçant les étapes de la politique pharmaceutique de l'indépendance aux installations d'usines de médicaments au Mozambique, force est de constater l'importance, à chaque période, des relations des professionnels de santé mozambicains avec des institutions et experts étrangers. Ces derniers sont responsables de l'introduction des compétences technologiques et de gestion nécessaires pour installer la production de médicaments. Dans tous les exemples, il s'agit de la mise en place d'industries de génériques relativement peu complexes et sans le développement de capacités de recherche ou de production des ingrédients pharmaceutiques actifs. La première usine de produits pharmaceutiques du pays installée par l'UNIDO ou les industries privées des années 1990 n'ont pas abouti à une accumulation d'un apprentissage technologique local. Si le développement de ces capacités était prévu dans le projet d'EMOFAR (1981-1988), les contraintes économiques conjoncturelles l'ont interrompu. Le cas de la coopération mozambico-brésilienne à la SMM se distingue par sa durée (2003-actuel) et la place majeure accordée à la formation et maîtrise des compétences de cadres locaux pour inclure les capacités d'investissement et de production. Si ce projet vise à terme l'autonomie locale de l'usine, il reste très dépendant du financement et des savoirs apportés par les acteurs brésiliens, fragilisant la possibilité de pérennisation de l'entreprise et d'accumulation des capacités. L'arrivée récente d'une filiale de Strides, grande firme de génériques indienne, relève d'un modèle tourné vers l'exportation et au sein duquel les compétences principales d'ingénierie de production sont la prérogative d'experts indiens, à priori sans transfert vers des cadres locaux. En somme, ces deux initiatives, montrent que si l'industrie pharmaceutique peut être crée par la circulation de savoirs technologiques et organisationnels transmis par des experts étrangers et acquis par des Mozambicains au Brésil ou en Inde, l'accumulation de ces savoirs pour maintenir la production à long terme dépend notamment du soutien et de la création de conditions favorables, économiques et de régulation, par les pouvoirs publics, dans un contexte où l'État a le quasi-monopole de la demande de médicaments.

Bien qu'au départ les acteurs de santé mozambicains aient pensé la fabrication locale comme moyen de garantir l'accès universel aux médicaments pour la population, au fil des années, de la transition vers l'économie capitaliste et le changement des générations, la priorité est passée aux investissements directs étrangers susceptibles de 
contribuer à la balance commerciale. L'expérience de plus d'une décennie pour la mise en place de la SMM, avec de nombreuses contraintes bureaucratiques, semble avoir convaincu certains acteurs du MISAU qu'il serait plus efficace de laisser les acteurs privés se charger de la production locale, sans forcément développer de stratégie pour soutenir ces derniers. Le MISAU semble se désengager de l'initiative de création de l'industrie pharmaceutique d'état, compromettant ainsi les chances de succès de la SMM. En coordination avec le Ministère de l'Industrie, il serait possible toutefois d'aligner les politiques d'accès aux médicaments et de promotion industrielle (Chaudhuri, Mackintosh et Mujinja, 2010). La comparaison des pays africains ayant des industries pharmaceutiques plus anciennes et qui subissent également la concurrence des firmes de génériques indiennes illustrent les possibilités qu'ont les gouvernements pour soutenir leurs industries locales de santé. Au Kenya, les industries pharmaceutiques ont pu augmenter leurs capacités pour répondre à la mise en place d'une politique «d'achat local » dans la décennie 1990, par laquelle le Ministère de la Santé déterminait que $50 \%$ des médicaments composants les kits sanitaires devaient être fabriqués localement (Mackintosh et al., 2016). Lorsqu'en 2003 le gouvernement kényan a annoncé qu'il mettrait en place le traitement contre le sida, les industries pharmaceutiques du pays se sont empressées de développer des versions génériques des ARV, mais seule une firme obtiendra, grâce au soutien de la coopération allemande, la certification OMS pour accéder aux marchés de bailleurs de fonds internationaux (Chorev, 2019). Si l'industrie pharmaceutique kényane continue de vendre ses médicaments génériques au gouvernement kényan (hors achats des bailleurs de fonds) et exporte aux pays voisins, Quet (2018) souligne qu'elle peut encore être davantage soutenue par des politiques améliorant l'environnement financier et les taxes sur l'importation de technologie (machines) ou des matières premières.

En outre, on constate une absence de mémoire institutionnelle au Mozambique, les quelques pharmaciens mozambicains connaissant l'histoire d'EMOFAR sont ceux actifs depuis les années 1980. Il serait intéressant de faire des recherches plus approfondies sur les trajectoires de ces professionnels, notamment ceux ayant été formés à l'étranger ou ayant pris part aux différentes initiatives de production locale. Cela permettrait de mieux comprendre pourquoi l'apprentissage à partir des expériences précédentes ou celle d'autres pays est quasi inexistant. Comprendre l'évolution de celles-ci pourrait fournir des leçons importantes pour mieux coordonner les politiques industrielles et de santé et favoriser l'accumulation de compétences pour la production locale de médicaments.

\section{BIBLIOGRAPHIE}

Abdenur A., Marcondes D., 2017, « Brazilian South-South cooperation in public health: Dilemmas of the Arv factory initiative ", dans A lden C., Chichava S., Alves A.C. (dirs.), Mozambique and Brazil: Forging New Partnerships or Developing Dependency? , Johannesburg, Jacana, p. 173-193.

Adamugy B., 1992, « Muitos doentes, poucos medicamentos », Domingo , 23 août 1992, p. 6-11. 
Almeida C., Campos R.P. De, Buss P., Ferreira J.R., Fonseca L.E., 2010, « A concepção brasileira de “cooperação Sul-Sul estruturante em saúde" ", Reciis , 4 , 1, p. 25-35.

Araujo S., Duarte R.G., Castro J.M. De , 2017, « Transferência de conhecimento na cooperação internacional : o caso Farmanguinhos - S MM », Revista de Saúde Pública , 51 , 103, p. 1-9.

Arvanitis R., Villavicencio D., Wei Z., 2014, « L'apprentissage technologique dans les pays émergents ", Revue d'anthropologie des connaissances , 8 , 3, p. 495-521.

Assemblée Mondiale De La Santé 28, 1975, «Vingt-huitième Assemblée mondiale de la Santé, Genève, 13-30 mai 1975 : partie I i : comptes rendus in extenso des séances plénières: procèsverbaux et rapports des commissions ", Genève.

Banda G., Wangwe S., Mackintosh M., 2016, « Making Medicines in Africa: An Historical Political Economy Overview », dans M ackintosh M., Banda G., Tibandebage P., Wamae W. (dirs.), Making Medicines in Africa , Palgrave Macmillan, p. 7-24.

Barbier A., 2014, « Sida : démarrage difficile pour l'usine publique d'antirétroviraux au Mozambique », Jeune Afrique , 14 octobre 2014.

Barker C., 1983, « The Mozambique Pharmaceutical Policy », The Lancet , 322 , 8353, p. 780-782.

Barker C., Marzagão C., Segall M., 1980, « Economy in drug prescribing in Mozambique », Tropical Doctor , 10 , p. 42-45.

Brasemb Maputo, 2002, «Telegrama Brasemb Maputo para Exteriores em 16/09/2002 n ${ }^{\circ} 521$. "Brasil-Moçambique. Cooperação na área da saúde. Construção de fábrica de retrovirais" ", Maputo.

Brasemb Maputo, 2003a, « Telegrama Brasemb Maputo para Exteriores em 05/02/2003 n ${ }^{\circ} 66$. "Brasil -Moçambique. CTPD . HIV/Aids. Programa de cooperação bilateral.” ", Maputo.

Brasemb Maputo, 2003b, « Telegrama de Brasemb Maputo para Exteriores em 25/07/2003 n ${ }^{\circ}$ 445. "Brasil-Moçambique. Visita presidencial, cooperação em DST/AIDS. Projeto da fábrica/ laboratório de Arvs. Missão do Ministério da Saúde. ", Maputo.

Brasil/Moçambique, 2001, « Protocolo de Intenções entre o Governo da República Federativa do Brasil e o Governo da República de Moçambique sobre Cooperação Técnica na Area da Saúde. Assinado em 19/06/2001. », p. 35.

Brasil/Moçambique, 2003, « Protocolo de Intenções entre o Governo da República Federativa do Brasil e o Governo da República de Moçambique sobre Cooperação Científica e Tecnológica na Área da Saúde. Assinado em 05/11/2003. ", p. 3.

Cahen M., 1987, Mozambique, la révolution implosée: Études sur 12 ans d'indépendace (1975-1987) , Paris, L'Harmattan, $170 \mathrm{p}$.

Cardoso F.H., 2000, « Discurso do senhor presidente da república, Fernando Henrique Cardoso, na III Conferência de Chefes de Estado e de Governo da Comunidade de Países de Língua portuguesa (CPLP), Maputo, Moçambique, 17 de julho de 2000. ", Resenha de Política Exterior do Brasil , 87,2 。 semestre de 2000, p. 27-30.

Cassier M., 2019, « La fin du partage? Les capitalismes de la copie face au capitalisme de la rente globale: une nouvelle géographie des industries de santé », Mouvements , 2 , 98, p. 107-119.

Chaudhuri S., Mackintosh M., Mujinja P.G.M., 2010, « Indian generics producers, access to essential medicines and local production in Africa: an argument with reference to Tanzania », European Journal of Development Research , 22 , 4, p. 451-468. 
Chorev N., 2019, « Making Medicines in Kenya, Tanzania, and Uganda in the A ids Era », Sociology of Development , 5, 2, p. 115-146.

Colin J.-P., 1976, «Le Mozambique un an après l'indépendance », Politique étrangère , 41 , 5, p. 433-458.

Esteves P., Assunção M., 2017, « South-South Partnership Puzzle: The Brazilian Health Expert Community in Mozambique », dans Bergamaschi I.,Moore P., Tickner A.B. (dirs.), South-South Cooperation Beyond the Myths. Rising Donors, New Aid Practices? , London, Palgrave Macmillan, p. 107-135.

Fiocruz, 2007, « Estudo de viabilidade técnico-econômico para instalação de fábrica de medicamentos em Moçambique para produção de medicamentos anti-retrovirais e outros », Rio de Janeiro.

Flynn M., 2013, « Brazilian pharmaceutical diplomacy : social democratic principles versus soft power interests », International Journal of Health Services , 43 , 1, p. 67-89.

Graber N., 2016, « Les biotechnologies cubaines sous l'angle des circulations globales : chercheurs rouges et diplomatie scientifique ", Cahiers de l'Urmis [En ligne] , 16 , p. 0-16.

Greene J.A., 2011, « Making medicines essential: The emergent centrality of pharmaceuticals in global health », BioSocieties , 6 , 1, p. 10-33.

Hanlon J., 1983, « Les médicaments essentiels au Mozambique », Santé du Monde , juin 1983, p. 26-29.

Hanlon J., 1985, « Beggars Can Be Choosers », New Scientist , janvier 1985, p. 7.

Hervieu S., 2011, « Le Mozambique va produire des antirétroviraux », Le Monde , avril 2011, p. 4.

Kovacs M., 1983, «Establishment of an oral rehydration salts production unit in Mozambique Dp/ Moz/80/017 », Budapest.

Lall S., 1992, « Technological Capabilities and Industrialization », World Development , 20 , 2, p. $165-186$

Lima T.G.F. De M.S., 2017, Cooperação internacional do Brasil em H iv/Aids: institucionalização e especificidades nos contextos nacional e internacional, Thèse de doctorat, Fundação Oswaldo Cruz, $320 \mathrm{p}$.

Luz F.C.S., 2006, A Questão da AIDS na África Austral: Realidade e Perspectivas da Cooperação Brasileira com os Países da Região para o Combate à Pandemia, Thèse de doctorat, Dissertação apresentada no âmbito do $50^{\circ}$ Curso de Altos Estudos do Instituto Rio Branco. Brasília: Ministério das Relações Exteriores.

Mackintosh M., Banda G., Tibandebage P., Wamae W., 2016, Making Medicines in Africa. The Political Economy of Industrializing for Local Health , $1^{\text {re }}$ édition, Palgrave Macmillan, $334 \mathrm{p}$.

Martins H., 1983, « Pharmaceutical Policy in Independent Mozambique: the first years ", The IDS Bulletin , 14,4 , p. $62-70$.

Martins H., 1985, « Uma Política farmacêutica de tipo novo », Revista de Administração publica , 19 , 1, p. $147-157$

Marzagão C., Segall M., 1983, « Drug selection: Mozambique », World Development , 11 , 3 , p. 205-216.

Moçambique, 2001, « Ministério da Saúde. Diploma Ministerial nº 183-A/2001, de 18 de Dezembro. ». 
Moçambique F. De Libertação De, 1977, Troisième Congrès du FRELIMO : 3-7 février 1977. Directives économiques et sociales , Paris, L'Harmattan, $63 \mathrm{p}$.

Mozambique R.P., 1979, Les soins de santé primaire au Mozambique, $1^{\text {re }}$ édition, Paris, L'Harmattan, $128 \mathrm{p}$.

Notícias, 2003, « Anti-retrovirais serão produzidos no país », Notícias , 6 novembre 2003, p. 1.

Oms, 1979, « Trente-deuxième Assemblée Mondiale de la Santé. Comptes rendus in extenso des séances plénières. Wha 32/1979/Rec/2 », Genève.

Oms Conseil Exécutif, 1978, « Programme d'action concernant les médicaments essentiels Eb $63 / 19 »$, Genève.

Patel S.J., 1983, "Third World Initiatives on Pharmaceuticals: a documentation for the record », The IDS Bulletin , 14 , 4, p. 71-78.

Pavignani E., Durão J.R., 1999, « Managing external resources in Mozambique: building new aid relationships on shifting sands? », Health Policy and Planning , 14 , 3, p. 243-253.

Pfeiffer J., 2013, « The Struggle for a Public Sector: PEPFAR in Mozambique », dans João Biehl, Adriana Petryna (dirs.), When People Come First: Critical Studies in Global Health , $1{ }^{\text {re }}$ édition, Princeton, Princeton University Press, p. 166-181.

Pitcher M.A., 2002, Tranforming Mozambique. The politics of privatization, 1975-2000 , Cambridge, Cambridge University Press, 293 p.

Porto De Oliveira O., Faria C.A.P. De, 2017, « Policy Transfer, Diffusion, and Circulation. Research Traditions and the State of the Discipline in Brazil », Novos estudos CEBRAP , 36 , 1, p. 13-32.

Pourraz J., 2019, Réguler et produire les médicaments contre le paludisme au Ghana et au Bénin : une affaire d'État? , Thèse de doctorat, École des Hautes Études en Sciences Sociales, Paris, France., $404 \mathrm{p}$.

Quet M., 2018, Impostures pharmaceutiques. Médicaments illicites et luttes pour l'accès à la santé. , Paris, La Découverte, $241 \mathrm{p}$.

Raj K., 2013, « Beyond postcolonialism... and postpositivism: Circulation and the global history of science », Isis , 104,2 , p. 337-347.

Rodrigues R.D., 2014, Cooperação Internacional da Fiocruz : O Caso do Projeto de Instalação da Fábrica de Medicamentos em Moçambique, Thèse de doctorat, Dissertação de Mestrado Profissional em Saúde Pública. Escola Nacional de Saúde Pública Sergio Arouca-Fiocruz. Rio de Janeiro, 197 p.

Russo G., Banda G., 2015, « Re-Thinking Pharmaceutical Production in Africa; Insights from the Analysis of the Local Manufacturing Dynamics in Mozambique and Zimbabwe », Studies in Comparative International Development , 50 , 2, p. 258-281.

Russo G., Oliveira L. De, 2016, « South-South Collaboration in Pharmaceuticals: Manufacturing Anti-retroviral Medicines in Mozambique », dans Mackintosh M., Banda G., Tibandebage P., Wamae W. (dirs.), Making Medicines in Africa, Palgrave Macmillan, p. 85-102.

Russo G., Oliveira L. De, Shankland A., Sitoe T., 2014, " On the margins of aid orthodoxy: the Brazil-Mozambique collaboration to produce essential medicines in Africa », Globalization and Health , 10,70 .

Ruxin J.N., 1994, « Magic bullet: The history of oral rehydration therapy », Medical History , 38 , 4 , p. 363-397. 
Scheinberg G., Bouer J., 2000, « País ajudará na produção de remédio », Folha de São Paulo , 11 juillet 2000, p. C6.

Unctad, 2011, «Local Production of Pharmaceuticals and Related Technology Transfer in Developing Countries », Geneva.

Unido, 1988a, « Technical Report: Potentials, Conditions and Parameters for Developing Pharmaceutical Industry in Mozambique. DP/MOZ/83/004. DP/ID/SER.A/1091 », Vienna.

Unido, 1988b, « Preparatory Assistance for the Establishment of a Pilot Plant For Pharmaceuticals. Terminal Report. Dp/Moz/83/004. Dp/Id/Ser.B/636 », Vienna.

Velásquez G., 1983, L'Industrie du médicament et le Tiers monde , 1 re édition, Paris, L'Harmattan, $207 \mathrm{p}$.

Velásquez G., 1985, Pour une politique du médicament: l'expérience du Mozambique , 1 re édition, Paris, l'Harmattan, $94 \mathrm{p}$.

Verschuur C., Lima M.C., Lamy P., Velásquez G., 1986, Mozambique: Dix ans de solitude... , 1 re édition, Paris, l'Harmattan, $182 \mathrm{p}$.

Walt G., Cliff J., 1986, « The dynamics of health policies in Mozambique 1975-85 », Health Policy and Planning , 1, 2, p. 148-157.

Who E.C., 1977, « The selection of essential drugs », Geneva.

World Bank , 1990, « Mozambique - Population, health and nutrition sector report ", Washington, Dc.

\section{NOTES}

1. Les ADPIC ont été introduits en 1994 par l'OMC et contraignent les gouvernements signataires à accorder dans leur législation nationale la protection de la propriété intellectuelle aux produits pharmaceutiques, entre autres. Cela permet aux firmes multinationales détentrices de brevets de conserver leur monopole et de pratiquer des prix élevés sur certaines molécules et procédés, notamment des médicaments contre le VIH/Sida. L'adoption de flexibilités des ADPIC pour des situations d'urgence sanitaire lors de la déclaration de Doha en 2001, le délai de dix ans pour son adoption dans les législations nationales et l'exemption de son adoption pour les pays les moins avancés exemption pour l'instant toujours prolongée - ont permis à divers pays de mettre en œuvre des stratégies industrielles et de santé publique permettant de fabriquer des médicaments génériques sur leur territoire.

2. Je remercie les évaluateurs pour leur relecture critique et les précieux conseils, ainsi que mes directeurs Maurice Cassier, Marilena Correa et les doctorants du Cermes3 pour leurs retours sur le papier en cours de rédaction. Cette recherche a été financée par une allocation doctorale de la Région Île-de-France et un financement Jeunes Chercheurs de Sidaction.

3. Suite à l'indépendance du Mozambique et au départ de colons portugais qualifiés, de nombreux étrangers, la plupart des personnes adhérant aux idées socialistes, ont répondu aux appels du gouvernement mozambicain pour faire venir des cadres qualifiés, des médecins en particulier, pour aider à construire le pays, G. Velásquez et C. Barker en faisaient partie (Colin 1976, discours. H, Martins à la WHA 31, 1978). 
4. L'expression Tiers-monde désignait dans l'après-guerre les pays n'appartenant ni au bloc occidental capitaliste, ni au bloc communiste, organisés après la Conférence de Bandung en 1955 au sein du Mouvement des Non Alignés. Dans la décennie 1970, ces pays - forts de leur majorité à l'Assemblée Générale de l'ONU suite aux indépendances des pays colonisés en Afrique et en Asie - ont investi les organisations onusiennes pour demander un Nouvel Ordre Économique International avec des principes répondant davantage aux besoins des pays en développement plutôt qu'aux intérêts des blocs dominants. L'influence des idées des Non Alignés et de leur poids fut un des moteurs du programme «Santé pour tous en l'an 2000 » de l'OMS, dont les politiques pharmaceutiques visant l'autonomie étaient une composante fondamentale (voir Nitsan Chorev, 2013, The World Health Organization between North and South, et Patel, 1983, op. cit .). Avec la fin de la guerre froide et les changements dans le mode de financement des actions internationales en santé, les pays non alignés ont perdu de leur influence dans ce domaine.

5. Le Comecon était une organisation économique regroupant les pays du Bloc de l'Est ayant pour objectif de planifier leurs économies et de répartir les spécialisations des industries.

6. Dans les années 1990, les principaux bailleurs de fonds, appelés «donateurs » au Mozambique, pour l'achat de médicaments au Mozambique, par le financement du Ministère de la Santé ou le don en nature, étaient les Agences de Coopération Canadienne, Danoise, Hollandaise, Norvégienne et Suisse (Pavignani et Durão, 1999).

7. Données disponibles sur https://www.who.int/3by5/en/Mozambique.pdf (consulté le $26 / 10 / 2019$ )

8. Le Mozambique ne serait pas le premier pays ayant une telle situation de maigres ressources financières et souhaitant investir dans la production locale. Cuba en dépit d'une forte crise économique et de pénuries alimentaires, suite à la chute de l'URSS et au renforcement de l'embargo nord-américain, a toutefois continué d'investir et de développer sa recherche dans le secteur des biotechnologies et son application dans les soins contre le cancer dans le système national de santé (Graber, 2016).

9. Le Mozambique a reçu par ailleurs du soutien de nombreux pays et organisations, notamment la Clinton Health Access Initiative, l'ONG Sant'Egidio, MSF et Health Alliance International. Une des caractéristiques de l'aide internationale pour le VIH/ sida au Mozambique est que le gouvernement a exigé que les initiatives prennent place au sein du service national de santé pour éviter l'érosion de ce dernier par des dispositifs de santé parallèles (Pfeiffer, 2013).

10. La crise politique au Brésil, associée à une crise économique en cours depuis 2014, aboutira à la destitution de la présidente Dilma Rousseff en 2016, qui avait succédé à Lula et appartenant au même Parti des Travailleurs (centre-gauche). Les financements et le fonctionnement de la coopération brésilienne pour l'installation de l'usine ont été perturbés pendant environ un an, jusqu'à ce que le gouvernement du vice-président Michel Temer (centre-droite) renouvelle le financement en 2017. Le Mozambique, qui était un "donor darling » avec son économie en forte expansion lors de la décennie précédente, révèle en avril 2016 des dettes contractées par l'État, probablement illégales, occultées aux donateurs et au FMI et subi depuis une crise économique liée à l'absence de confiance des institutions internationales (voir «Le Mozambique dans l'enfer de la dette» par Adrien Barbier, Le Monde, 22/12/2018 https:// 
www.lemonde.fr/economie/article/2018/12/22/le-mozambique-dans-l-enfer-de-ladette_5401422_3234.html).

\section{RÉSUMÉS}

À son indépendance en 1975, le Mozambique affichait une politique volontariste d'autonomie en médicaments essentiels. Cependant, les diverses initiatives d'autonomisation avec l'aide internationale dans la mise en place de la production locale de médicaments n'ont pas abouti dans les années 1980. Le gouvernement acquiert en 2008 une usine pharmaceutique privée en faillite pour recevoir un transfert de technologie et de compétences des laboratoires du Ministère de la Santé du Brésil, qui finance les équipements et la formation des cadres et techniciens mozambicains par leurs pairs brésiliens. La résilience économique de cette usine publique - la Sociedade Moçambicana de Medicamentos - est mise à l'épreuve par la concurrence des importations via des bailleurs de fonds internationaux de médicaments génériques fabriqués en Inde et, plus récemment, l'installation d'une usine d'un laboratoire privé indien au Mozambique. À travers des entretiens, observations et archives sur les expériences de production locale au Mozambique, cet article décrit les dynamiques de transfert de connaissances et compétences dans le domaine de la pharmacie industrielle entre les professionnels mozambicains et internationaux. Ce faisant, nous analyserons l'évolution du médicament comme bien public dans le pays, entre accessibilité et qualité pour la santé publique et rendements pour l'industrie.

In the first years after its independence in 1975, Mozambique had a voluntarist pharmaceutical policy that prioritised essential generic medicines, in accordance with the World Health Organization's policies at the time of 'Health for All' and the Alma-Ata Conference. Several initiatives to install local production of medicines in Mozambique were started in the late 1970s1980s with International support from United Nations Agencies, bilateral cooperation from European and Socialist countries and a Swedish multinational pharmaceutical company. UNIDO experts from Hungary and Bangladesh did install an Oral Rehydration Salts production unit in Beira City, funded by PNUD. The EMOFAR plant (Mozambique Pharmaceutical Enterprise, in its Portuguese acronym) did not manage to produce at full capacity in a context of economic crisis, structural adjustment, civil war and lack of professionals with the requirements to be trained for its management and production process and closed around 1989.

In 2008, the Mozambican government acquired a bankrupted private pharmaceutical serum plant in order to receive a transfer of technology and skills training from Brazilian Ministry of Health State owned laboratory Farmanguinhos. The new Mozambican factory, named Mozambican Society of Medicines (SMM in its Portuguese acronym), was first conceived to produce antiretrovirals for HIV/AIDS treatment, but Mozambican health authorities negotiated with their Brazilian counterparts to include other essential medicines in its portfolio. By including common antidiabetic, hypertension and analgesic drugs, Mozambican health officials linked this SouthSouth Cooperation project with their 1970s pharmaceutical policies centred on the Essential Medicines List. The Brazilian government funded the equipment, the training and the raw materials. The training of Mozambican technicians and managers by a team of Brazilian pharmacists, chemists and engineers with extensive work experience in private-owned pharmaceutical plants in Brazil, took place in Mozambique and in Brazil. The transfer of knowledge and skills and building capabilities is ongoing since 2008, with phases of theoretical 
and practical (on-the-job) training. The participants stressed that the technological learning process also encompassed the acquisition of managerial skills and of international certification norms such as Good Manufacturing Practices. This process revealed the challenges of transferring knowledge between two asymmetrical personnel, in terms of initial training and work experience. The retention of trained Mozambican professionals in a context with limited qualified professionals is also problematic.

Moreover, some Mozambican authorities and their Brazilian partners still debate the aims of this enterprise: to produce generic drugs exclusively for the Ministry of Health supply or to export production to generate revenue for the Mozambican State. The economic resiliency of SMM in put under strain by the competition of medicine imports from international donors, such as PEPFAR, and the low prices from Indian Generic Manufacturers. More recently, an Indian Generic Pharmaceutical Firm installed a new plant only minutes away from SMM, also training Mozambican staff in India, and is already selling some of its production to the Mozambican Ministry of Health. It was found, however, that this firm did not promote local acquisition of managerial and more complex technical skills for their Mozambican staff, limiting the possibility of autonomy should the foreign staff leave. In a context where the State is responsible for the majority of medicines procurement, the Ministry of Health and the Ministry of Industry and Commerce have yet to formulate a policy for the two pharmaceutical plants installed in the country or to include them in their strategic plans.

The case of the EMOFAR factory in the 1980s and the more recent SMM project and Indian private subsidiary show that Mozambique's political willingness to foster local production of medicines is highly dependent on capital, technological and managerial transfers and circulation from foreign actors. Although the Mozambican health authorities did sometimes adapt the knowledge and objectives of these enterprises into their priorities, so far, they have failed to support the plants in the long term with specific health, fiscal or industrial policies. This situation thus questions whether the status of medicines in Mozambique, which were regarded as strategic public goods that required local production for economic, political and access to health reasons, is changing.

This article draws on work for a PhD thesis that describes the dynamics of knowledge and skills transfer in industrial pharmacy between Mozambican and international professionals, focusing especially on the SMM project. The methodology included interviews, on site observations at the SMM plant and the gathering of official documents and archives on past local production experiences in Mozambique.

\section{INDEX}

Mots-clés : production locale, médicaments génériques, circulation de savoirs

Keywords : local production, generic medicines, knowledge circulation

Index géographique : Mozambique

\section{AUTEUR}

\section{ALILA BROSSARD ANTONIELLI}

École des Hautes Études en Sciences Sociales - EHESS Centre de recherche médecine, sciences, santé, santé mentale, société - CERMES3 